\title{
Giant collisions involving young Jupiter
}

\author{
A. Anic, Y. Alibert, and W. Benz
}

\begin{abstract}
Physikalisches Institut, University of Bern, Sidlerstrasse 5, 3012 Bern, Switzerland
e-mail: augustin.anic@space.unibe.ch
\end{abstract}

Received 6 June 2006 / Accepted 29 November 2006

\section{ABSTRACT}

\begin{abstract}
We present high-resolution, three-dimensional simulations using a smooth particle hydrodynamics (SPH) code of giant impacts involving young Jupiter-like planets. Our aim is to explore the effect of such impacts on the structure and evolution of the planet and discuss the likelihood of detecting these post-impact planets. For this, we considered head-on and off-axis impacts by an Earth-like planet onto a young Jupiter at five different ages: $1 \mathrm{Myr}, 10 \mathrm{Myr}, 30 \mathrm{Myr}, 100 \mathrm{Myr}$, and $1 \mathrm{Gyr}$. We briefly discuss the short-term post-impact evolution and concentrate on computing the long-term cooling of the planet. We find that the bright IR afterglow lasts for about $10^{6} \mathrm{yr}$ if the impact involves a 1 Myr old planet and up to $10^{8} \mathrm{yr}$ if the impact occurs on an older planet ( 30 Myr). We estimate that, about 10 to 100 young planetary systems must be observed to detect one candidate for such post-impact object. Given that their luminosity is only increased by a roughly $50 \%$, this frequency does not make them ideal observing targets. We note nevertheless that the detection of this kind of post giant-impact planet would represent an important milestone in observationally establishing the current planet formation theories that are based on collisions.
\end{abstract}

Key words. stars: planetary systems - stars: planetary systems: formation - solar system: formation

\section{Introduction}

The standard model for planetary-system formation suggests that a significant number of giant impacts may occur during the last stages of planets formation (Wetherill 1990; Tonks et al. 1990). These events presumably lead to the ejection of Mercury's mantle (Benz et al. 1988), the formation of the EarthMoon system (Wetherill 1985; Benz et al. 1986); Stevenson (1987), and the Pluto-Charon system (Stern 1992; Canup 2005). While most of the attention has been devoted to giant impacts involving terrestrial planets, these events may involve ice or gas giants as well, if they are a generic characteristics of planet formation, and may be the origin of planetary obliquities (see Lissauer \& Safronov 1991; and references therein). Indeed, the large spin obliquity of Uranus (98 degree) is attributed to a strong tangential collision with another protoplanet at the end of the accretion process (e.g. Korycansky et al. 1990; Slaterry et al. 1992; Parisi \& Brunini 1997). Saturn may owe its obliquity (27 degrees) to an impact by a very large protoplanet (Lissauer \& Safronov 1991; Parisi \& Brunini 2002)

Stern (1994) considered the impact of Earth-like planets onto various giant planets as a way of increasing their intrinsic luminosity, thus making them considerably easier to detect. He estimated the thermal flux from an impacted Uranus-like planet located in the nearest star forming region some $135 \mathrm{pc}$ away (a typical distance for nearby star forming regions such as Taurus-Auriga and $\rho$ Ophiuchus) and found that the detection of such an object would not only be possible but would also require only about one hour observing time using a Keck-class telescope. However, even if these impacted planets may be quite luminous, the likelihood of actually observing them is directly proportional to the time during which they remain super-luminous. This timescale, coupled with the frequency of giant impacts occurring in a typical planetary system, can be used to estimate how many systems have to be observed in order to detect one post-impact, luminous young planet (Zhang \& Sigurdsson 2003, hereafter ZS03).

The detection of an impact-induced super luminous planet would represent an important milestone in establishing the existence of giant impacts observationally and thus of the entire framework of planetary growth through collisions that predict these events. It is therefore quite important to compute the detection probability as accurately as possible. In the abovementioned studies, the effect of a giant impact on the structure, hence on the expected luminosity change of the planet, has been estimated from first principle. In this paper, we improve on this by actually simulating a number of such impacts using a 3D smooth particles hydrodynamics (SPH) code (Benz 1990) and computing the expected post-impact structure of the planet. We then evolve the planet until it again becomes indistinguishable from a reference, non-impacted planet. This allows us to quantify the changes in the planet's structure as a function of time. In particular, we address the actual increase in detectability and discuss whether these events can account for the discrepancy between the observationally derived and the calculated radius of some of the transiting EGPs such as HD 209458b (Cody \& Sasselov 2002).

The paper is organized in four sections. In Sect. 2 we briefly present our numerical approach including the SPH collision simulation code and the equations used to calculate the long-term evolution of impacted planets. We also briefly discuss the equations of state (EOS) used in our calculations. In Sect. 3, we analyze the results of the $3 \mathrm{D}$ collision calculations, compute the energy deposition inside the impacted planet, and describe the expected electromagnetic (EM) signal accompanying such an event. The long-term evolution of the impacted planets, as well as the detectability of these objects, are computed in Sect. 4. Finally, we summarize and discuss the results in Sect. 5. 


\section{Numerical models}

\subsection{Computational technique}

Calculations are performed in two stages. In the first stage we simulate giant collisions between Earth-sized projectiles and various young giant planets of a Jupiter mass using a 3D SPH code invoking over 200000 particles ${ }^{1}$. This method has been extensively described in the literature (see e.g. Benz 1990; Steinmetz \& Müller 1992) and will not be discussed here again. Let us simply mention that in our calculations a $\beta$-spline kernel is used (Monaghan \& Latanzio 1985) and that density and internal energy (not entropy) are chosen as the independent thermodynamic variables in SPH equations, whereby the density of each particle is calculated by summing the contributions from the density profile of neighboring particles. Finally, for the artificial viscosity we use the time varying form proposed by Morris \& Monaghan (1997). In all the following SPH calculations, the conservation of the total energy has proved to be very good (variation in total energy was $\leq 1 \%$ ).

We carry out these calculations for as long as possible until the planets have recovered a nearly spherical shape. This allows us, in the second stage, to map the resulting structure into a 1D planetary evolution code to compute their long term evolution. For this purpose we solve the following equations

$$
\begin{aligned}
\frac{\mathrm{d} r^{3}}{\mathrm{~d} m} & =\frac{3}{4 \pi \rho} \\
\frac{\mathrm{d} L}{\mathrm{~d} m} & =-\frac{\mathrm{d} U}{\mathrm{~d} t}+\frac{P}{\rho^{2}} \frac{\mathrm{d} \rho}{\mathrm{d} t} \\
\frac{\mathrm{d} P}{\mathrm{~d} m} & =\frac{-G\left(m+M_{\text {core }}\right)}{4 \pi r^{4}} \\
\frac{\mathrm{d} T}{\mathrm{~d} P} & =\nabla_{\mathrm{ad}} \text { or } \nabla_{\mathrm{rad}}
\end{aligned}
$$

$r, L, P, T, \rho$ and $U$ are the radius, the luminosity, the pressure, the temperature, the density, and the internal energy, all functions of $m$, the amount of mass enclosed inside a sphere of radius $r$. The opacities are taken from Alexander \& Fergusson (1994), and the equation of state (EOS) from Saumon et al. (1995).

The temperature gradient is equal to the adiabatic one $\nabla=$ $\nabla_{\text {ad }}$ or the radiative one $\nabla=\nabla_{\text {rad }}$ depending on the stability of the zone against convection, which is given by the Schwarzschild criterion. The outer boundary conditions for this calculation are taken from Allard et al. (2001), at an optical depth of $\tau=100$. These boundary conditions relate the pressure $P$ and temperature $T$, at $\tau=100$, to the effective temperature $T_{\text {eff }}$ and luminosity $L$ of the planet. We do not take the effect of the irradiation of the parent star into account.

\subsection{Equation of state}

To model the various components of target and projectile, we use different EOS. The projectile is assumed to be made of two layers: a core made of iron $(\mathrm{Fe})$ and a mantle made of dunite $\left(\mathrm{Mg}_{2} \mathrm{SiO}_{4}\right)$. The mass ratio of these two components is 0.5 . The material properties of the projectile are calculated using the ANEOS EOS (Thompson \& Lauson 1984). Details on this EOS can be found elsewhere (e.g. Benz et al. 1989; Canup 2004).

\footnotetext{
1 When constructing 3D bodies, we set masses of the SPH particles in such a way that the density profile in $3 \mathrm{D}$ matches the one in $1 \mathrm{D}$, which obviously leads to particles of different masses. The mass variation between edge and center of the planet is proportional to the density variation.
}

The gaseous envelope of the giant planet is assumed to be composed of hydrogen and helium and is calculated using the Saumon et al. (1995) EOS in both stages of the calculation, 3D impact and 1D long-term evolution. The helium mass fraction is equal to 0.25 , and we do not take into account some heavy elements inside the envelope.

Since the exact size of Jupiter's core is unknown, we considered planets with a core and planets without. Guillot et al. (1994) argue that the core is composed of rocks enriched by some $30 \%$ of ice. However, as we shall see below, the calculations show that the outcome of a collision is quite insensitive to the presence or absence of the core and that its exact nature turns out to be irrelevant for our purpose. Therefore, to save computer time, we model the core using the simple Tillotson EOS (Melosh 1989) assuming dunite represents the rocky material.

\subsection{Initial conditions and early evolution of the target}

Since the current modeling of Jupiter's structure (e.g. Saumon \& Guillot 2004) does not allow the size of the planet's core to be determined, we have considered two cases, one without a core - labeled "J" in the following and another one with a core labeled "JC". The mass of the core itself is taken to be $15 M_{\oplus}$, close to the upper limit derived by Saumon \& Guillot (2004). In this way we believe that our two models bracket all possible core sizes and should allow us to determine its potential effect on the collision.

The initial structure of the target is calculated using our 1D evolution code in the following way. The total mass of the planet is set equal to the mass of present-day Jupiter $\left(1 M_{\mathrm{J}}\right)$ including the core, if any. The radius of the planet is less well-constrained since we are actually dealing with a young planet (giant collisions happen during the early stages of a planetary system). To compute this radius, we proceed by setting it to an arbitrarily high value and let the planet evolve for a long timescale (typically $1 \mathrm{Myr}$ ) compared to its Kelvin-Helmholtz time in order for it to forget its initial conditions. At that time, our young Jupiter has an effective temperature of $T_{\text {eff }}=1033 \mathrm{~K}$, a mass of $1 M_{\mathrm{J}}$, and a radius on the order of $1.7 R_{\mathrm{J}}$, where $R_{\mathrm{J}}$ is the present day-radius of Jupiter. However, the precise value depends on the presence of a core. The corresponding thermodynamical conditions at $\tau=100$ (the outer radius for our 1D calculation) are: $P_{0} \sim 4.92 \times 10^{6} \mathrm{dyn}^{-2}$ and $T_{0} \sim 2600 \mathrm{~K}$. The resulting temperature, density, and pressure profiles for both models, $\mathrm{J}$ and JC, are presented in Figs. 1-3, respectively. The radius of model $\mathrm{J}, R_{\mathrm{yJ}}=1.28 \times 10^{10} \mathrm{~cm}$ (radius of young Jupiter), is somewhat larger than the radius of model JC, $R_{\mathrm{yJC}}=1.25 \times 10^{10} \mathrm{~cm}$ (radius of young Jupiter with a core). Note in Fig. 2 that the density profile for model JC is discontinuous at the core/envelope interface.

Since the time of the collision is actually unknown and since the structure of the planet is fairly rapidly changing early on, we also compute the structure for older planets, using our 1D evolution code. For this purpose, we evolve the 1D profile for $\mathrm{J}$ (Jupiter without a core) to $10 \mathrm{Myr}, 30 \mathrm{Myr}, 100 \mathrm{Myr}$, and $1 \mathrm{Gyr}$ and use the resulting structures to study the effect of a giant impact at an increasingly later time. To illustrate the changes in the internal structure of the planet as a function of age, we show temperature-pressure profiles in Fig. 4 (a possible core is not considered) and provide the corresponding radius in Table 1 for each of these older planets.

The giant collisions we envision occur most probably during the early epoch of the formation of the planetary system at a time when collisions involve large bodies. Considering the 


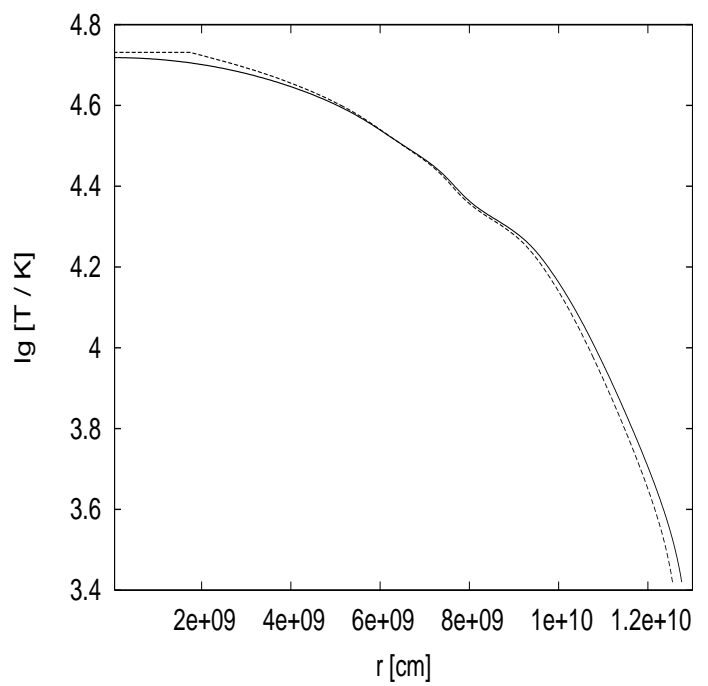

Fig. 1. Temperature profiles for models $\mathrm{J}$ (solid line) and JC (dashed line). The core in model JC is assumed to be isothermal.

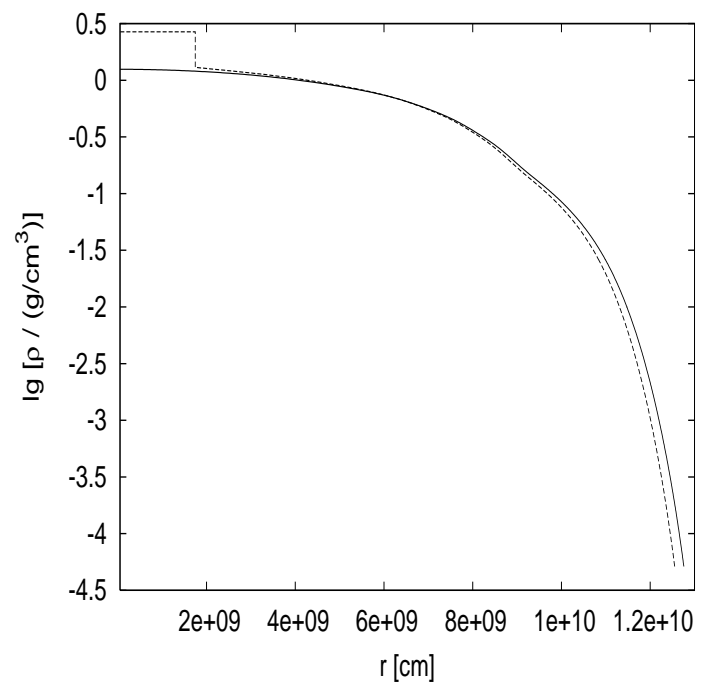

Fig. 2. Density profiles for models J and JC. The meaning of the lines is as in Fig. 1. The jump in model JC is located at the core/envelope interface.

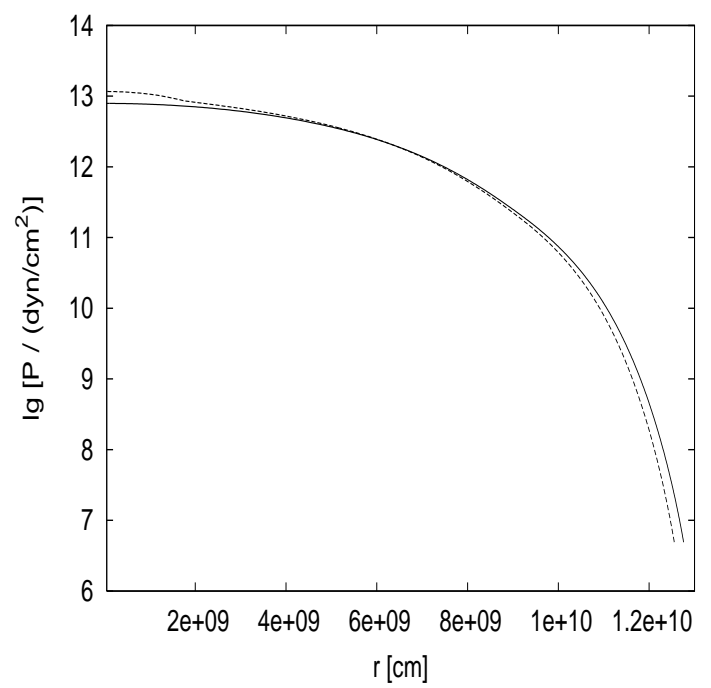

Fig. 3. Same as Fig. 1, for the pressure.

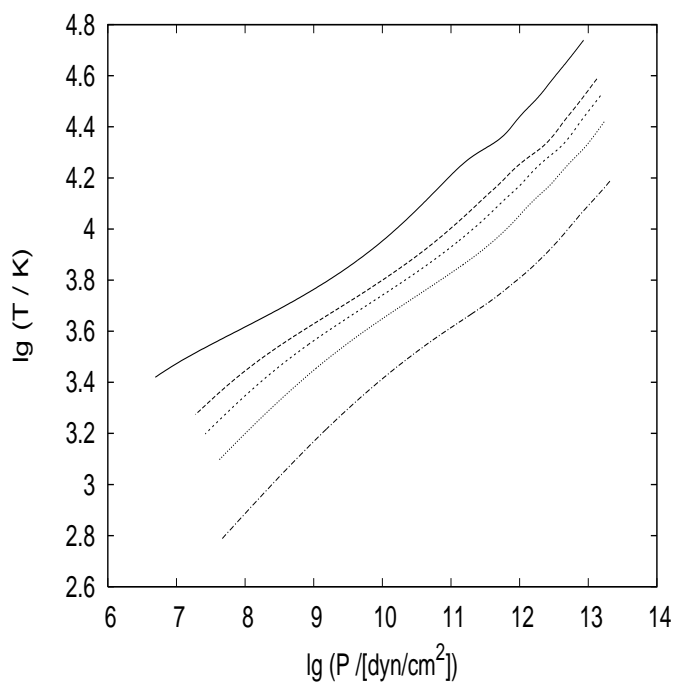

Fig. 4. Temperature-pressure profiles for differently aged Jupiter. From bottom to top: a $1 \mathrm{Gyr}, 100 \mathrm{Myr}, 30 \mathrm{Myr}, 10 \mathrm{Myr}$, and a $1 \mathrm{Myr}$ old Jupiter.

Table 1. Radii at different epochs in the evolution of Jupiter.

\begin{tabular}{cc}
\hline \hline$R[\mathrm{~cm}]$ & $t[\mathrm{Myr}]$ \\
\hline $9.55 \times 10^{9}$ & 10 \\
$8.98 \times 10^{9}$ & 30 \\
$8.38 \times 10^{9}$ & 100 \\
$7.61 \times 10^{9}$ & 1000 \\
\hline
\end{tabular}

epoch, it is reasonable to assume that the projectile is probably molten. The mass of the projectile is unknown: giant impacts are likely to involve projectiles of very different masses. However, typical $N$-body simulations show that the ratio between the masses of the two more massive colliding bodies is roughly one order of magnitude (Wetherill 1985). Taking the mass of Jupiter's core to be $\sim 10 M_{\oplus}$, we therefore adopt a projectile of $1 M_{\oplus}$. This choice also allows comparisons with the work of ZS03. Pritchard \& Stevenson (2000) calculated the surface temperatures of Earth-sized bodies involved in collisions and suggest temperature around $2000 \mathrm{~K}$. Canup (2004) generated similar bodies by impacting a dunite object with an iron projectile and derived temperatures of about $4000 \mathrm{~K}$. Hence, for lack of better constraints, we adopt a completely molten projectile with a surface temperature of $2600 \mathrm{~K}$. We neglect the potential presence of an atmosphere and set the surface pressure to zero. With these boundary conditions and assuming a total mass equal to $M_{\mathrm{P}}=1 M_{\oplus}$, we can calculate the internal structure of the planet by integrating the structure equation from outside inwards, which leads to a final radius of $R_{\mathrm{P}}=6.53 \times 10^{8} \mathrm{~cm}$.

Using these $1 \mathrm{D}$ profiles for the planet and the projectile, we generate the $3 \mathrm{D}$ bodies which are used in the SPH simulations. The initial distance between target and projectile is set to 1.1 times the target radius. Four collision simulations involving the very young (1 Myr) Jupiter are carried out. The first two, SIM1 and SIM2, involve the target J (core-less Jupiter) while the other two, SIM3 and SIM4, involve target JC (Jupiter with a core). SIM1 and SIM3 are head-on collisions, while SIM2 and SIM4 are off-axis collisions with impact parameters $b=0.5 R_{\mathrm{yJ}}$ and $b=0.5 R_{\mathrm{yJC}}$, respectively. The initial relative velocity is set to the escape velocity from the surface of the planet, which is $v_{\text {rel }}=45.6 \mathrm{~km} \mathrm{~s}^{-1}$ for SIM1 to SIM4. 
Table 2. From left to right: simulation number, age of the target, relative velocity, and impact parameter given in units of the corresponding planet's radius (see Table 1).

\begin{tabular}{ccccc}
\hline \hline SIM\# & $t[\mathrm{Myr}]$ & $v_{\text {rel }}\left[\mathrm{km} \mathrm{s}^{-1}\right]$ & $b[\mathrm{R}]$ & $M_{\text {core }} / M_{\oplus}$ \\
\hline SIM1 & 1 & 45.6 & 0 & 0 \\
SIM2 & 1 & 45.6 & 0.5 & 0 \\
SIM3 & 1 & 45.6 & 0 & 15 \\
SIM4 & 1 & 45.6 & 0.5 & 15 \\
SIM5 & 10 & 52.74 & 0.5 & 0 \\
SIM6 & 30 & 54.41 & 0.5 & 0 \\
SIM7 & 100 & 56.31 & 0.5 & 0 \\
SIM8 & 1000 & 59.11 & 0.5 & 0 \\
\hline
\end{tabular}

Four additional collision simulations are carried out involving older target planets. These simulations are labeled SIM5, SIM6, SIM7, and SIM8, in increasing order of the age of the target planet. Table 2 provides a detailed list of all the initial conditions used in our simulations.

We carried out these calculations using slightly over 200000 particles to model both the target and the projectile. This resolution should be sufficient for determining the quantities required for our investigation. The simulations were carried out until the impacted planet recovered a spherical shape and most of the ejected, but still bound material, re-impacted the planet. Once we have reached this stage, we determine the energy deposition profile and the post-impact new internal structure, which we then map back into the 1D planetary evolution code for subsequent long-term evolution.

\section{Outcomes of collisions}

In this section, we analyze the 3D simulations and compare them to each other. We are primarily interested in computing the trajectory of the projectile inside the planet's envelope and the corresponding local energy deposition. This energy deposition leads to substantial temperature and entropy profile changes and thus to changes in the subsequent evolution of the planet. We start with a brief description of SIM1 (head-on collision onto 1 Myr Jupiter, without a core), which serves as a generic collision model. For this simulation, a total of $\sim 220000$ particles have been used with $\sim 650$ for modeling the projectile.

\subsection{Giant impacts onto a young Jupiter without a core}

\subsubsection{Trajectory of the projectile}

During the first stage of the impact the projectile penetrates the thin outer layers of the target. This part of the envelope is too thin to slow down the projectile, which keeps accelerating. About 18 minutes after entering the envelope, drag forces exceed the gravitational attraction and the projectile starts to slow down. At this stage, the projectile is located $5.2 \times 10^{9} \mathrm{~cm}$ beneath the surface; and the unperturbed pressure at this level is $P \sim 9.7 \times 10^{11}$ dyn $\mathrm{cm}^{-2}$. The ambient gas is compressed and a bow shock forms in front of the projectile. Instead of a pancaking effect, which would appear when small objects penetrate the atmosphere, we observe the collateral flattening of the projectile. This behavior has already been described by Korycansky et al. (2000), who shows that the mass loss of large bodies is due to mechanical ablation rather than thermal ablation. These authors show that hydrodynamic instabilities (Rayleigh-Taylor, Kelvin-Helmhotz) grow before compressional waves have time to cross the projectile. Thus, the rear side of the impactor is left mostly unperturbed, while the front side gets highly distorted. In the snapshots of SIM1 (Fig. 5, see in particular the second one), the flattening of the projectile and even the projectile's core (in red) can be clearly seen. Finally, during this phase of the impact, the mantle (in orange) is partially peeled off (see the snapshots 3 and 4).

In fact, the projectile is not stopped by the target and actually reaches the center after about one hour. After this, the projectile begins to oscillate about the center. While initially strong, these motions are damped over a few hours. By then, the target has still not recovered its spherical shape. We notice that some small fraction of material (less than $0.1 \%$ of the total mass) is accelerated during the collision beyond escape velocity and lost by the giant planet.

\subsubsection{Energy deposition inside the envelope}

During its journey through the planet's envelope, the projectile transfers its kinetic energy to the surrounding gas. We calculate the energy deposition profiles by differentiating the kinetic energy of the incoming projectile. More precisely, in the first phase of our calculation, when SPH code is used, we determine the kinetic energy $E$ of the projectile (or, better said, the loss of kinetic energy) with respect to the mass center of the target. Having calculated $E(r)$ for each time step of our SPH calculation ( $r$ denotes the distance from the target's mass center), the deposition profile $\mathrm{d} E / \mathrm{d} r$ follows by first-order numerical differentiation. The energy deposition rate profiles of SIM1 and SIM2 are compared in Fig. 6. The most efficient energy transfer in SIM1 occurs near the center of the target, whereas in SIM2 the peak occurs in a layer located between $0.25 R_{\mathrm{yJ}}$ and $0.4 R_{\mathrm{yJ}}$. Hence, in the offaxis collision the projectile transfers its kinetic energy most efficiently while traversing the median part of the envelope, whereas in the head-on collision the energy deposition rate is maximum near the center. However, it is important to note that most of the kinetic energy in the head-on collision is transferred before the projectile reaches the center of the target, as can be seen in Fig. 7, where we present the amount of energy dissipated along the trajectory of the projectile. In the same figure, one also may see that the location where most of the energy is stored is somewhat deeper in the case of SIM1 than in the case of SIM2.

A closer inspection of the trajectory of the projectiles explains the differences in the energy deposition rates of the discussed collision types. In the head-on case, the projectile reaches the center and begins a damped oscillatory motion about it, while in the off-axis case the projectiles spirals toward the center reaching it with almost zero velocity. This is to be expected because the deceleration is governed essentially by the mass encountered by the projectile, which is larger for a given depth below the surface in an off-axis collision. In other words, the projectile in SIM2 must plow through more envelope to reach the same depth as the projectile in SIM1, and thus kinetic energy deposition as a function of depth is more efficient in off-axis collisions. This kinetic energy is deposited in the form of heat, which gives rise to a growing plume rising toward the surface (see Takata et al. 1994, for detailed studies of this subject).

\subsubsection{Structure of the impacted planet}

Collisions are followed using the SPH code until the target has recovered a nearly spherical shape and all oscillations have been damped. At that time, we remap the $3 \mathrm{D}$ structure into a $1 \mathrm{D}$ structure in order to follow its evolution to late times. For this 

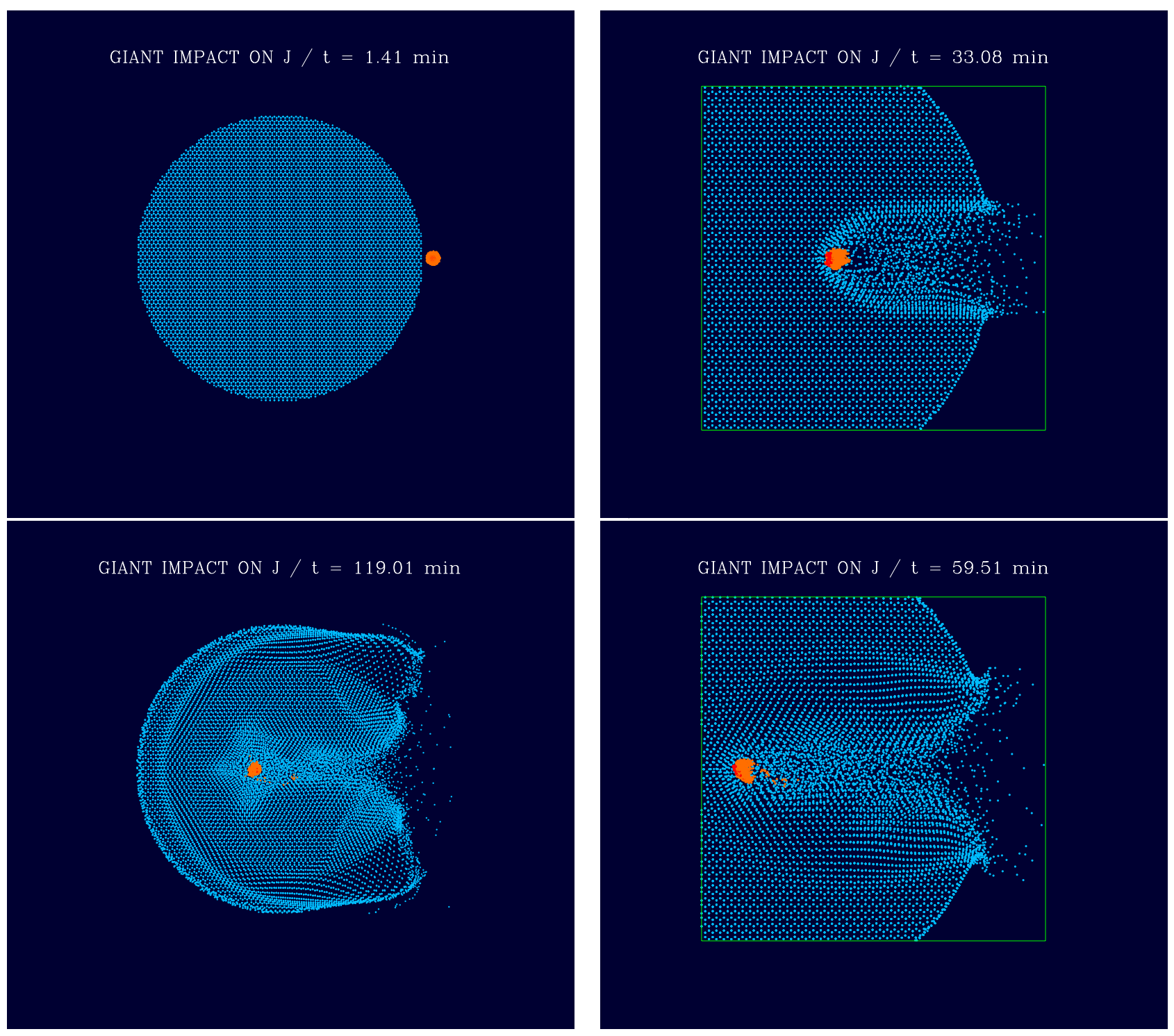

Fig. 5. Four snapshots of the head-on collision SIM1 covering about two hours of real time (ordered clockwise). Each is a narrow slice in the plane of the collision. The projectile consists of an iron core (red) and a silicate mantle (orange), which is partially lost due to mechanical ablation (second and third snapshots, which are magnified). Two final snapshots show the propagation of the shock wave through the envelope of the target.

mapping, we start from the center of mass of the impacted planet and bin particles in spherical shells until we reach at least $99.5 \%$ of the mass of the remaining planet. The distance between the last layer and the center gives the radius of the post-impact planet. Typically, the simulated impacts lead to a radius increase of about $\sim 18 \%$. During the time immediately following the impact, $\sim 2$ months (see the next section), the radius decreases, but is still 5\% larger at the end of this phase than before the impact. We have also found out that the total mass of the planet is nearly unchanged after the impact, but that there is a significant change in the heat content. The 1D profiles given in the next section supports these statements (see for example Fig. 8).

\subsubsection{Short-term evolution of the impacted planet}

After the projectile has settled in the center and the planet has roughly recovered its spherical shape, the subsequent evolution still proceeds relatively rapidly. During this phase, which we do not model, the energy released by the impact inside the envelope is redistributed in the planet, and the envelope reaches a state of constant specific entropy (ZS03). As shown by ZS03, the relevant timescale $\tau$ is given by $\tau \sim N_{\mathrm{c}} l / v_{\mathrm{c}}$, where $N_{\mathrm{c}}$ is the typical number of convective overturns needed to reach an almost uniform entropy inside the entire envelope, where $v_{\mathrm{c}}$ is the convective speed and the convective mixing length $l$ is defined as the depth where the velocity of the projectile is reduced to half of its original impact velocity. This length-scale is also the location where most of the energy due to the impact is stored. In our simulations, this length-scale is found to be $l \sim 0.55 R_{\mathrm{yJ}}$ for SIM1 (head-on collision) and $l \sim 0.4 R_{\mathrm{yJ}}$ for SIM2 (off-axis collision). We note that these values are higher than the ones given in ZS03, but, as shown by Canup \& Asphaug (2003), the stripping of the mantle from the projectile increases its penetration depth. Assuming further, as in ZSO3, $v_{\mathrm{c}} \sim 1 \mathrm{~km} \mathrm{~s}^{-1}$ and $N_{\mathrm{c}}=100$, we obtain $\tau \sim 60-80$ days depending on the geometry of the collision.

During this time, the energy deposited initially relatively deep in the envelope is redistributed throughout the entire envelope through convection. Radiative losses during this period are relatively modest, as this redistribution timescale $\tau$ is much 


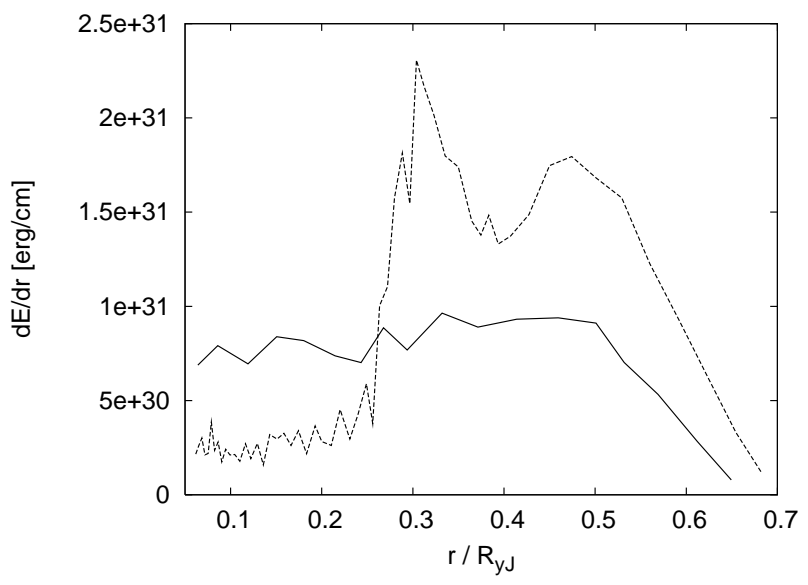

Fig. 6. Energy deposition rate (along trajectory) profiles for SIM1 (solid curve) and SIM2 (dashed curve). The peak in the dashed curve points to a rapid energy release.

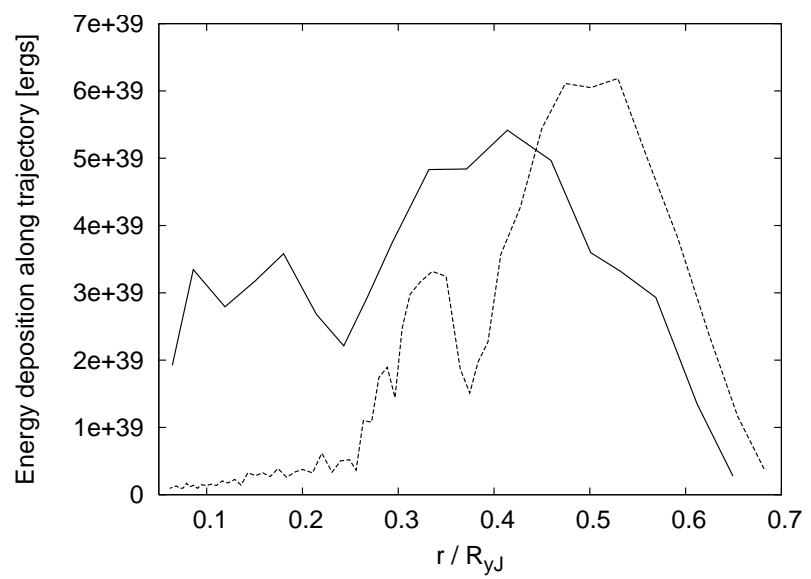

Fig. 7. Energy deposition (dE) profiles for SIM1 (solid curve) and SIM2 (dashed curve). The latter type of the impact (SIM2, off-axis collision) leads to a more efficient distribution of the deposited energy inside the envelope.

shorter than the time required by the planet to lose its internal energy content (see Sect. 4.1). We therefore assume that the entropy inside the envelope at the beginning of the long-term evolution is still equal to the one just after the impact. Furthermore, the specific entropy is uniform inside the whole gaseous envelope due to this convective redistribution process.

The temperature, pressure, and density profiles before and after the impact, as well as after the short-term evolution phase, are shown in Figs. 8-10. The dotted curves are steeper than those directly after the impact (dashed curves), and the radius is still greater than before the impact, due to the increase in the total entropy of the envelope.

\subsection{Giant impacts onto young Jupiter with a core}

We now briefly describe the outcome of simulations SIM3 and SIM4, calculated assuming a $15 M_{\oplus}$ core for the 1 Myr old Jupiter. These simulations are carried out with $\sim 200000$ particles, $\sim 2000$ of them inside the projectile and the core. The snapshots presented here are for SIM4, the off-axis collision.

The trajectories of the projectile from these simulations are very similar to the ones from the simulations with the Jupiter without a core. The projectile, once decelerated, is flattened laterally. As in the previous case, the impactor penetrates and starts

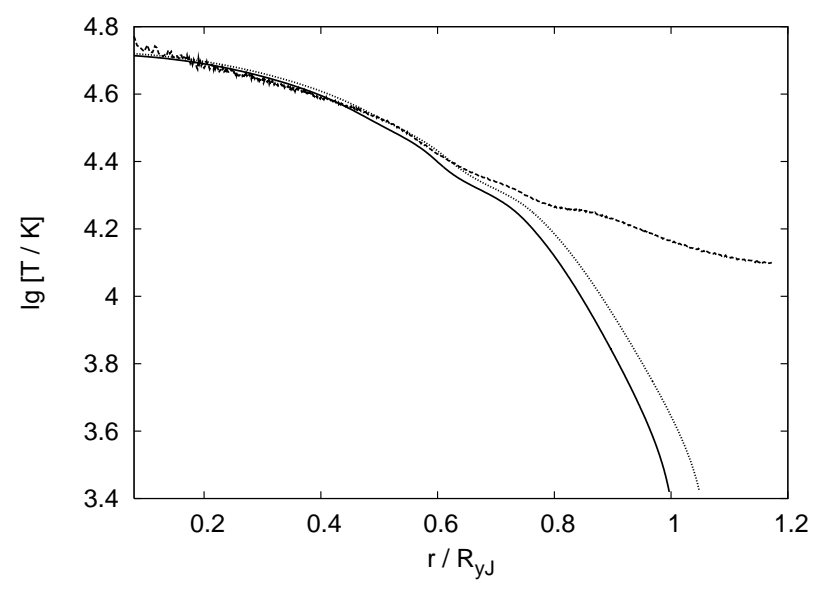

Fig. 8. Temperature profiles for the young Jupiter. From bottom to top: before the impact (solid curve), $\tau \sim 2$ months after the collision (dotted curve), and directly after the collision (dashed curve).

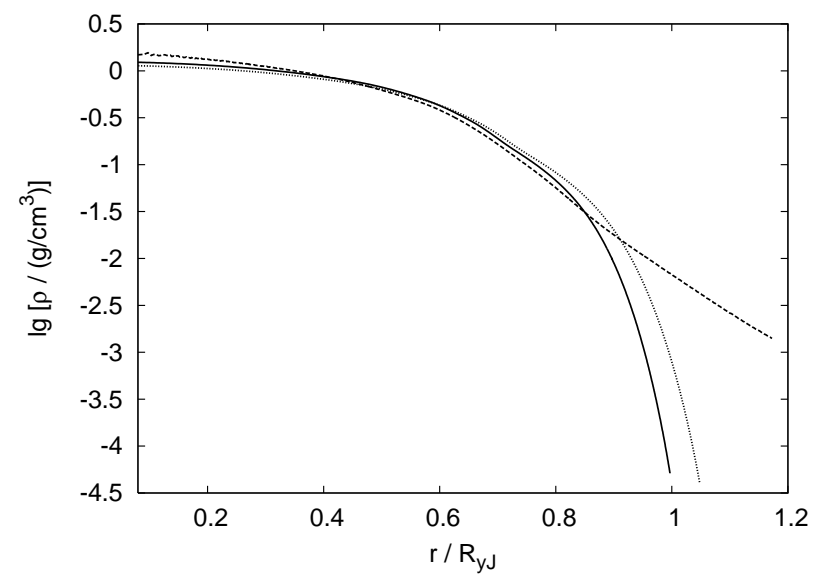

Fig. 9. Same as Fig. 8 for the density profiles.

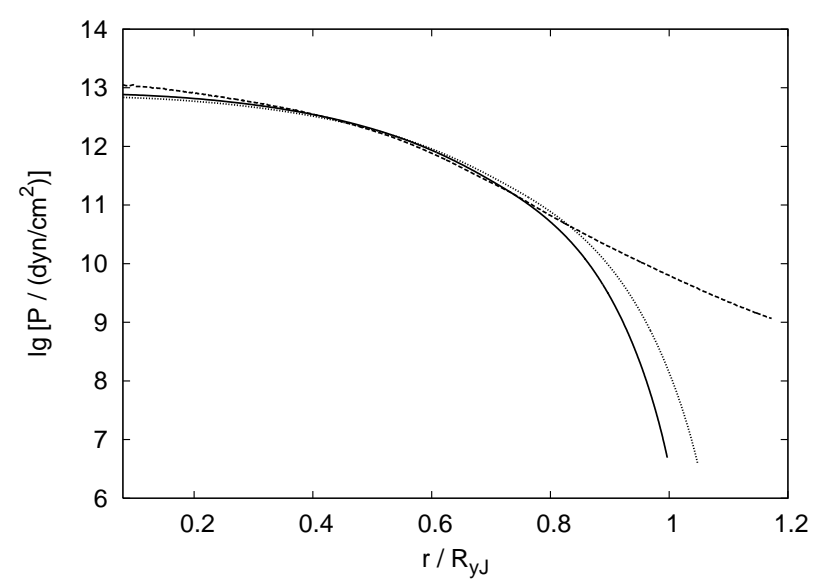

Fig. 10. Same as Fig. 8 for the pressure profiles.

to decelerate deeper in SIM3 (head-on collision, $4.3 \times 10^{9} \mathrm{~cm}$ below the surface, corresponding to $P \sim 4.6 \times 10^{11} \mathrm{dyn}^{-2}$ ) than in SIM4 (off-axis collision, $3.8 \times 10^{9} \mathrm{~cm}, P \sim 3.1 \times$

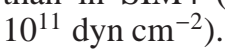

Figure 11 shows the projectile that spirals into the core of the target. When these two bodies meet, around two hours after the start of the simulation, the relative velocity of the projectile is still very high $\left(\geq 19 \mathrm{~km} \mathrm{~s}^{-1}\right)$ and a partial disruption of the two bodies is observed. The phenomena is seen very well in the 

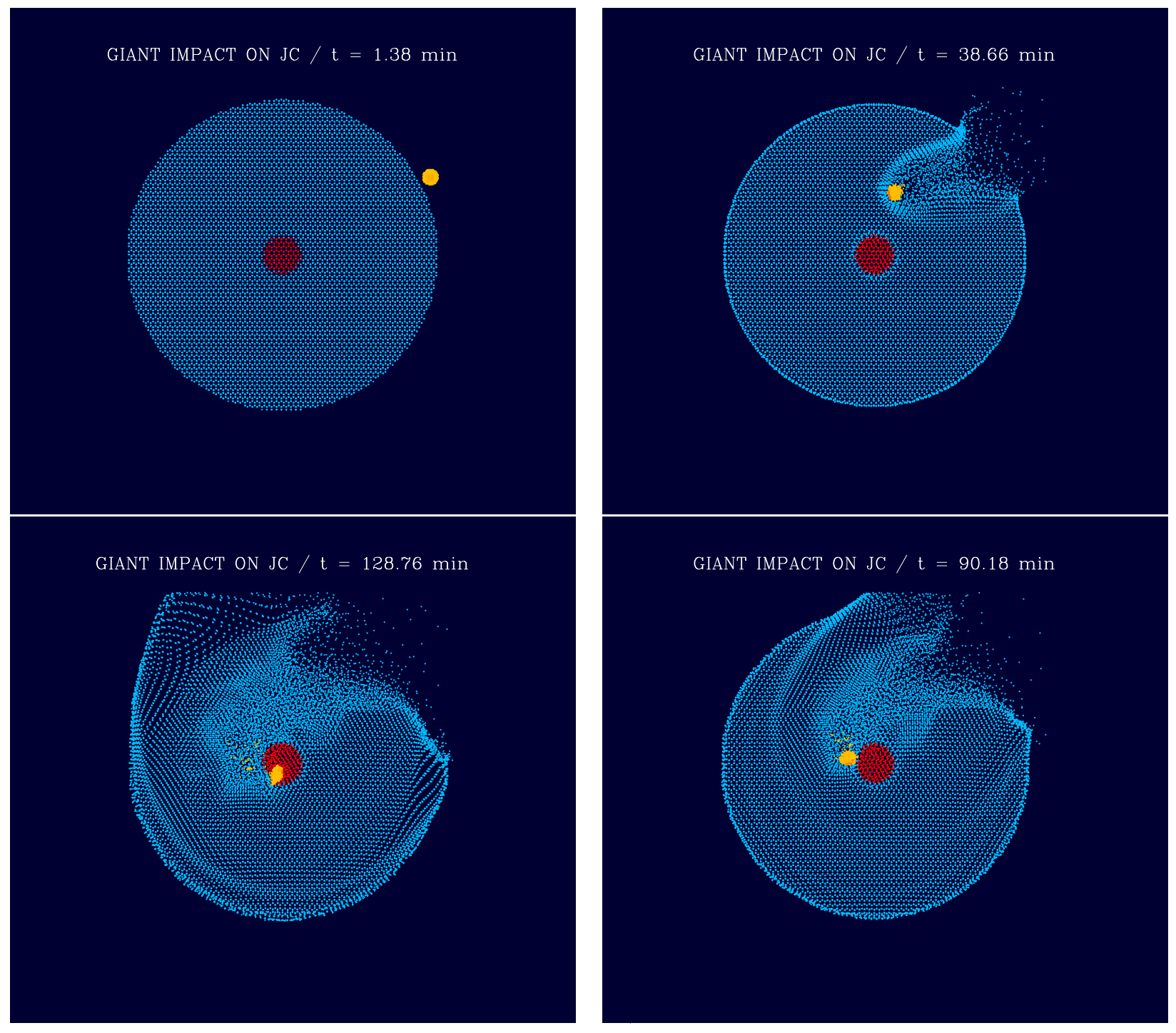

Fig. 11. Four snapshots of SIM4 covering about two hours of the real time collision history (ordered clockwise). Blue is for the envelope of the target, yellow for the impactor, whereas red is for the core of the target. The core of the impactor (in orange) is not recognizable until the hydrodynamic pressure reveals it (second and the third snapshot).

energy deposition profiles in Fig. 12. The curves are characterized by peaks at the center revealing the very rapid energy release from the projectile to the envelope of the target. Except for near the core, the energy in the two head-on collisions (SIM1 and SIM3) is deposited at very similar locations, compared to the two off-axis collisions (SIM2 and SIM4 - compare Figs. 6 and 12, and Figs. 7 and 13). The effect of the presence of the core is thus negligible compared to the effect of the impact geometry. This justifies the use of Tillotson EOS instead of ANEOS EOS (see Sect. 2.2) to calculate the structure of the core. Indeed, most of the planet's mass is inside its envelope, which controls both the outcome of the impact and the long-term evolution.

The increment in radius due to the off-axis collision (SIM4) is $\Delta R / R \sim 16 \%$ (calculated in the same fashion as in the previous section), slightly lower than in the head-on collision (SIM1), but it decreases more slowly during the short-term evolution, to $\triangle R / R \sim 7 \%$.

The $1 \mathrm{D}$ profiles for temperature, pressure, and density are calculated in the same way as in the previous section and are presented in Figs. 14-16, where we do not include the core in the calculation of the structure after the impact. To complete the discussion we show the differences in the 2D internal energy profiles between the pre- and the post-impact planet. The snapshots are given in Fig. 17 and reveal clearly that the post-impact planet is hotter than the pre-impact planet and that it, $\sim 3$ days after the impact, has almost recovered its spherical shape.

\subsection{Collision induced observable signals}

Since we do not explicitly model radiation in our SPH code, we cannot directly derive the signal expected from the collision. We therefore use the results of our simulations, together with the work of ZS03, to compute the expected signature. The reader is referred to ZS03 for further details. Since the effect of the core is quite small, we consider here only the simulations involving the 1 Myr old Jupiter without a core (simulations SIM1 and SIM2, the initial radius of the planet being, for both cases, $\left.R_{\mathrm{yJ}}\right)$. The impact geometry, on the other hand, has been shown to play a significant role and will be discussed in the section devoted to the long-term evolution below.

As shown by $\mathrm{ZSO3}$, the expected signal following the collision can be divided into four different phases, each with its 


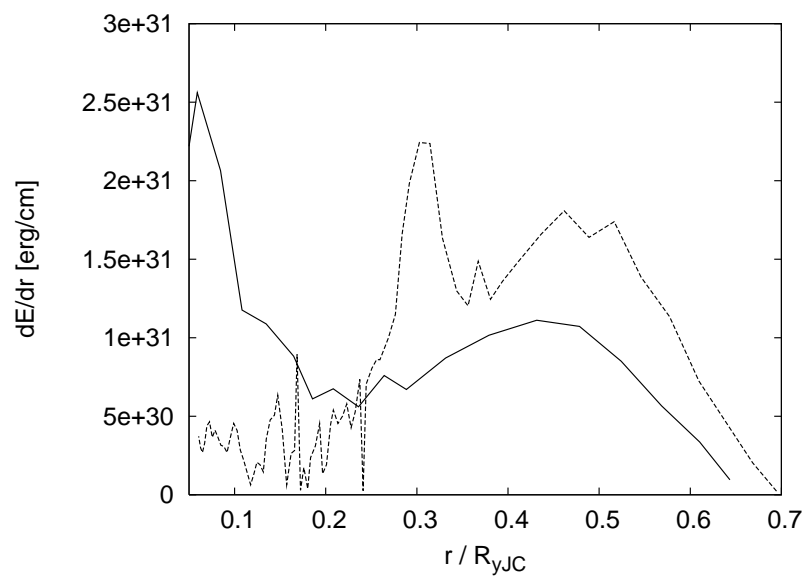

Fig. 12. Same as Fig. 6 for SIM3 (solid curve) and SIM4 (dashed curve).

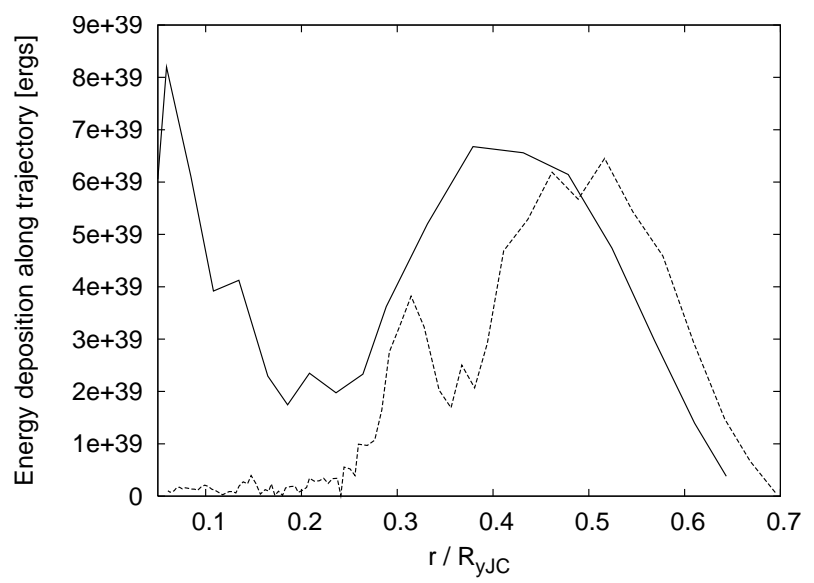

Fig. 13. Same as Fig. 7 for SIM3 (solid curve) and SIM4 (dashed curve).

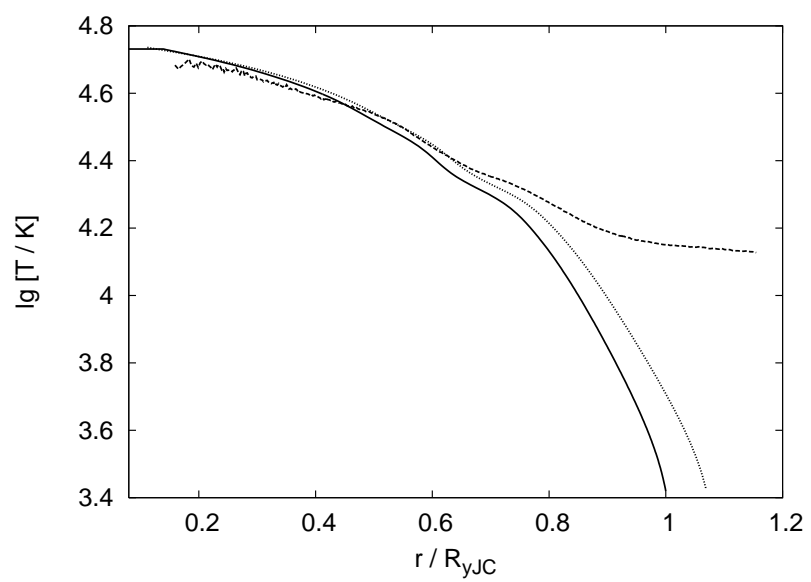

Fig. 14. Temperature profiles for Jupiter with a core. From bottom to top: before the impact, after the short time evolution, and directly after the impact. The lines have the same meaning as in Fig. 8.

specific timescale. The first phase is characterized by the penetration of the envelope by the projectile. Since the velocity of the latter is highly supersonic, a shock wave propagates both into the projectile and in the envelope. These shock waves convert part of the kinetic energy of the projectile into internal energy and generates the first flash. The rising time of this first flash is given by the time required by the shock wave to cross the projectile,

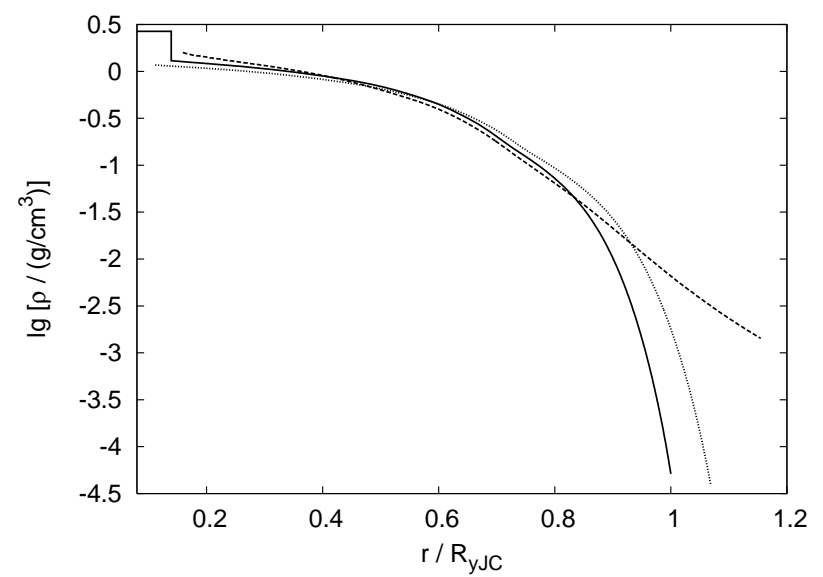

Fig. 15. Same as Fig. 14, for the density. Note that, after the impact, the core is no longer taken into account since it has no importance for the long-term evolution (see the absence of jumps in the curves at the core/envelope interface).

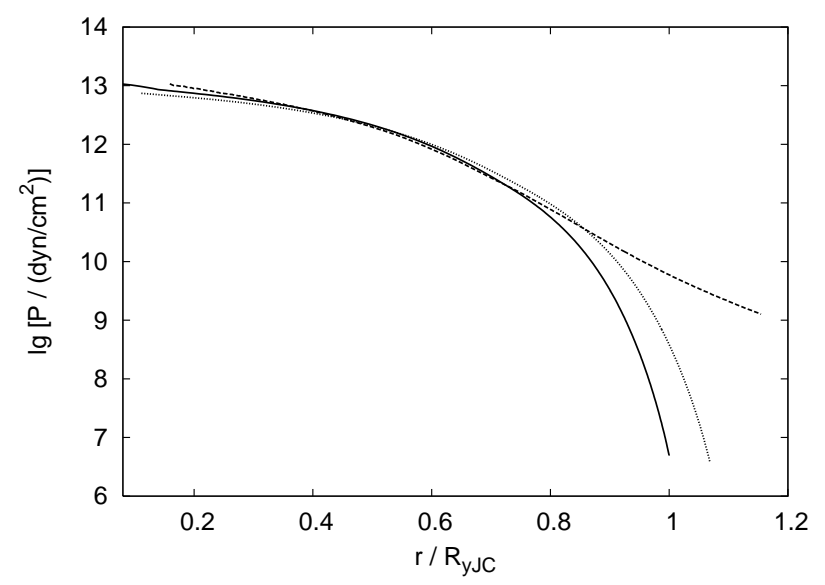

Fig. 16. Same as Fig. 14 for the pressure.

i.e. $\tau_{\mathrm{sc}} \sim R_{\mathrm{P}} / c_{\mathrm{s}} \sim 21 \mathrm{~min}$, where $c_{\mathrm{s}} \approx 5.2 \mathrm{~km} \mathrm{~s}^{-1}$ is the sound speed inside the projectile ${ }^{2}$, and $R_{\mathrm{P}}=6.53 \times 10^{8} \mathrm{~cm}$ is its radius.

The rate of energy deposition during the first phase, which is calculated using the first order approximation in our energy deposition profile, is $\mathrm{d} E / \mathrm{d} t \sim E / \tau_{\mathrm{sc}} \sim 3 \times 10^{37} \mathrm{ergs} \mathrm{s}^{-1}$, which is $\sim 8 \times 10^{4}$ time the Eddington luminosity of the young Jupiter $L_{\text {Edd }}=4.17 \times 10^{34} \mathrm{ergs} \mathrm{s}^{-1}$. According to ZS03, the photons are trapped during this prompt phase by the high opacity in the regions where heat is initially deposited, and the peak bolometric luminosity is given by $L_{\text {peak }}=\eta L_{\text {Edd }}$, where $\eta \leq 1$ is a factor for correcting less than optimal emission geometry and other radiative inefficiencies. Using the same consideration, we obtain $L_{\text {peak }} \sim 11 L_{\odot}$, where $L_{\odot}=3.8 \times 10^{33} \mathrm{ergs} \mathrm{s}^{-1}$ is the solar luminosity. During the second phase, the radiation pressure, which is induced by the impact, causes the photosphere of the target to expand. During the expansion, the photosphere cools and when the radiation pressure starts to decline, it falls back onto the "surface" of the planet which presumably leads to some electromagnetic (EM) flashes. ZS03 approximated the time scale of this phase by assuming that the emission luminosity is still above $50 \%$ of $L_{\text {peak }}$ at its end. We adopt the respective value, since we cannot estimate the duration with our computing

\footnotetext{
${ }^{2}$ We note that our value is lower than the one for Earth given by Dziewonski \& Anderson (1981), $c_{\mathrm{s}} \approx 10 \mathrm{~km} \mathrm{~s}^{-1}$, since the projectile considered here is much hotter.
} 

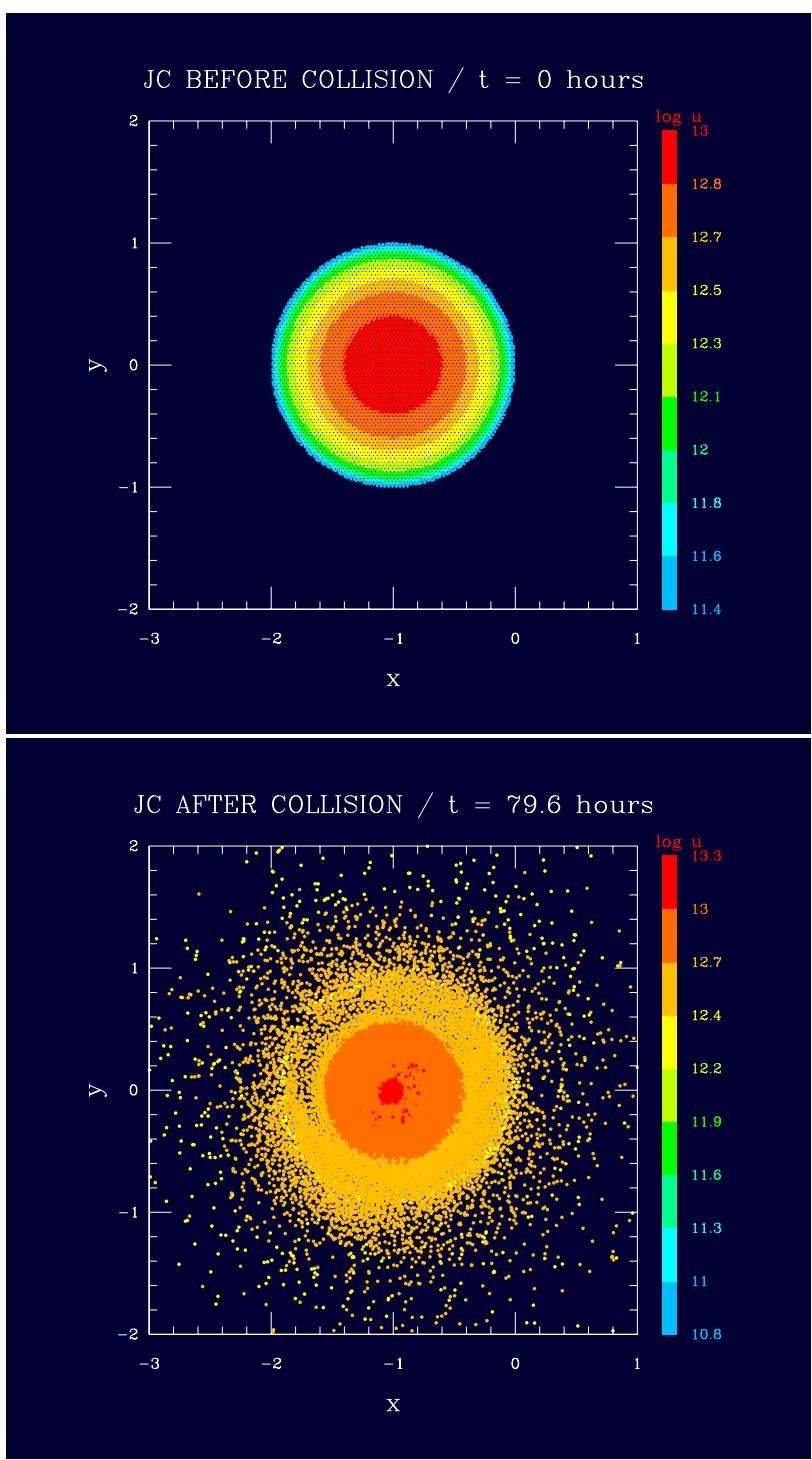

Fig. 17. Target before and after the collision in SIM4. The color bar on the right side yields the thermal energy of particles involved in the simulation. Coordinates of four corners are given in units of the radius of the pre-impact planet $-R_{\mathrm{yJC}}$.

model. The time scale proposed by the authors is $\tilde{\tau} \sim 10 \times \tau_{\text {sc }}$, and the total energy being radiated during this time period is $\tilde{E} \sim L_{\text {Edd }} \times \tilde{\tau} \sim 10^{38}$ erg or less then $1 \%$ of the impact energy.

The second phase ends when the hot spot starts to spread due to convection inside the planet, which we discussed in detail in Sect. 3.1.4. In the same section, we found out that this phase (the third in the list) lasts for about two to three months, after which the surface has reached an equilibrium temperature. Finally, the planet enters the last phase (the long-term evolution), during which the largest fraction by far of the impact energy is radiated away, the so-called IR afterglow.

\section{Long term evolution and detection of impacted planets}

The probability of detecting a post-impact planet depends essentially on three quantities: 1) the number of giant collisions occurring in a typical planetary system, $N_{\mathrm{p}} ; 2$ ) the total lifetime of the planetary system during which collisions do occur, $T_{\text {system }}$; and

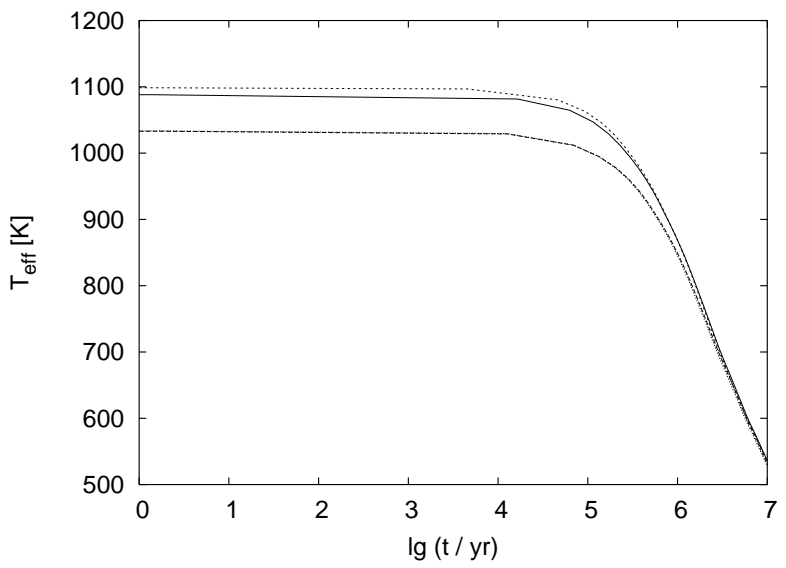

Fig. 18. Effective temperatures (in Kelvin) versus age (in years) for the reference and impacted giant planets. From bottom to top, first line: evolutionary sequence for the reference body. Two lines are actually drawn, one of them corresponding to J, the other to JC. The effect of the core is negligible as far as the evolution of the planet is concerned. Second line: SIM1 (head-on collision). Third line: SIM4 (off-axis collision).

3) the time during which an impacted planet remains more luminous than a normal reference planet having not suffered from an impact, $t_{\text {hot }}$. This last quantity may also depend on the conditions of the impact (e.g. geometry, structure of impacted planet). The first two quantities have been estimated by Stern (1994) and Wetherill (1990), and we shall use their results. The results of our collision simulations, together with the long term evolution calculation, provide the missing quantity, $t_{\text {hot }}$.

We can actually define $t_{\text {hot }}$ in two different ways. First, we can define it as the time required by the planet to radiate the entire kinetic energy of the projectile (which has actually been deposited inside the planet), $t_{\text {hot }, 1}$ :

$E_{\text {impact }}=\int_{0}^{t_{\text {hot, }, 1}} L(t) \mathrm{d} t$

where $L(t)$ is the time dependent luminosity of the post-impact planet (see Fig. 19). This definition is the one used by ZS03. However, we see that even though the planet has radiated the entire additional energy, it is still more luminous than an identical reference planet that did not suffer from an impact. Hence, we can define a second timescale, $t_{\text {hot, } 2}$, as the time required by the impacted planet to become indistinguishable (within a few percent) from a non-impacted planet. This timescale is then defined using the condition

$\frac{L_{\text {impacted }}\left(t_{\text {hot }, 2}\right)-L_{\text {non-impacted }}\left(t_{\text {hot }, 2}\right)}{L_{\text {non-impacted }}\left(t_{\text {hot }, 2}\right)} \leq 10 \%$.

Based on this condition, the impacted planet would no longer have any advantages over a non-impacted planet after $t_{\text {hot,2 }}$.

\subsection{Long-term evolution of post-impact EGPS}

In this section we calculate the time evolution of two giant planets. One is our reference planet, which does not suffer from an impact, while the other one is the impacted planet. Comparing both planets allows us to determine the impact of specific changes on the structure and hence the luminosity of the young planet. We consider two simulations, SIM1 and SIM4, i.e. one head-on collision and one off-axis collision, both assuming a 1 Myr old Jupiter. 


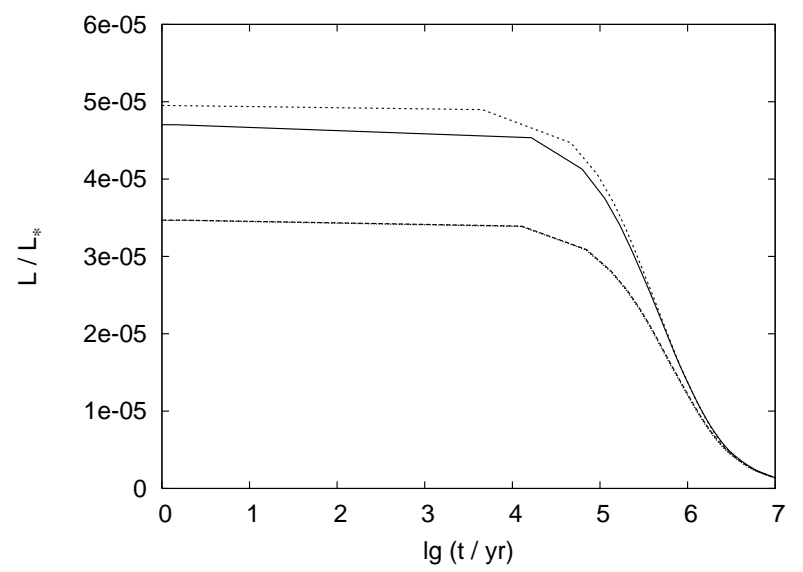

Fig. 19. Evolution of the luminosity (in solar units) versus age (in years), for the same models as in Fig. 18.

The resulting evolution of the effective temperature and the total luminosity are presented in Figs. 18 and 19. At the beginning of the evolution, the effective temperature for the reference models (with or without a core) is $1033 \mathrm{~K}$. This effective temperature increases to $1088 \mathrm{~K}$ for the planet suffering a head-on collision and to $1100 \mathrm{~K}$ for the one involved in an off-axis collision. It then takes about $10^{6}$ to $10^{7}$ years until the reference and post-impact planets become indistinguishable because they have the same surface temperature and total luminosity. From the plot it is also clear that the effective temperature actually starts noticeably to decrease about $10^{4}-10^{5} \mathrm{yr}$ after the impact. This confirms that the assumption of neglecting the radiation losses at early times was actually justified.

Using the results of our evolution calculations of the postimpact planet, we find a timescale for the IR afterglow of $t_{\text {hot, } 1}=$ $1.07 \times 10^{4} \mathrm{yr}$ for the head-on collision and $t_{\text {hot }, 1}=1.02 \times 10^{4} \mathrm{yr}$ for the off-axis collision. ZS03 obtains somewhat lower values, between $10^{3} \mathrm{yr}$ and $10^{4} \mathrm{yr}$. This difference is probably due to their assumption of a power-law decay for the luminosity from the start, whereas we find that the luminosity does not decrease markedly during the first $\sim 10^{4}$ yr. Furthermore, our calculations also predict a lower effective temperature than ZS03 (on the order of $1500 \mathrm{~K}-2500 \mathrm{~K}$, compared to less than $1100 \mathrm{~K}$ in our simulations); hence, radiation losses are considerably reduced.

Using our second definition of the timescale for the IR afterglow, we obtain $3.18 \times 10^{6} \mathrm{yr}$ and $5.86 \times 10^{6} \mathrm{yr}$. These numbers are typically two orders of magnitude higher than those obtained using the other definition. Thus, we conclude that giant impacts of the type envisioned here alter the structure of the planet considerably, a difference that lasts much longer than just the time needed to actually radiate the impact energy.

\subsection{Effects of the target's age}

Since the age of the planet at the time of the impact is essentially unknown, we carried out a set of simulations using planets with ages $10 \mathrm{Myr}, 30 \mathrm{Myr}, 100 \mathrm{Myr}$, and 1 Gyr. In all cases we proceeded in an identical fashion to what is described above: The impact proper was simulated using a 3D SPH code and the resulting structure was fed into a 1D planetary evolution code. To assess the effect of the collision we again computed the evolution of a corresponding reference planet that had not suffered from such an impact.

The results are presented in Fig. 20, where the luminosity versus time for the different age planets is shown. From this

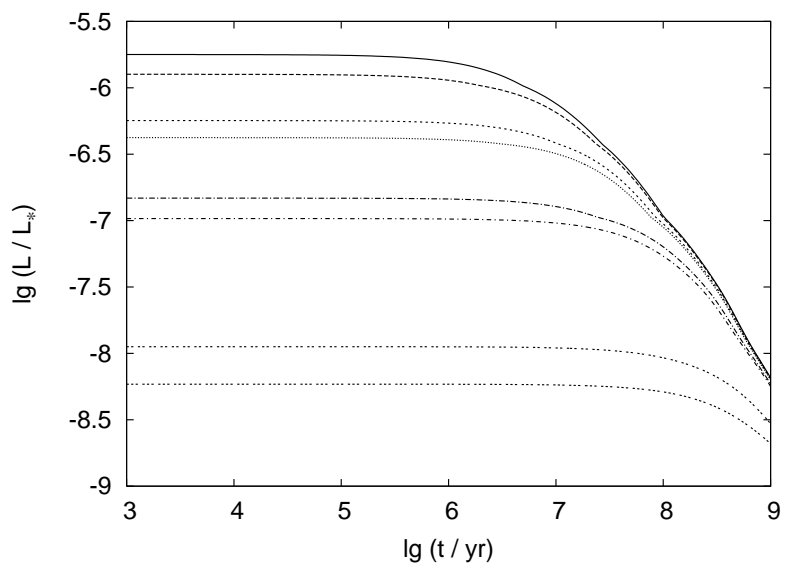

Fig. 20. Evolution of the luminosity (in solar units) versus age (in years), for planets of ages: $1 \mathrm{Gyr}, 100 \mathrm{Myr}, 30 \mathrm{Myr}, 10 \mathrm{Myr}$ (bottom to top). In each case, we show the evolution of both the reference and the impacted planet.

figure it is easy to see that the time at which the giant impact occurs matters in two aspects. First, the luminosity increase is higher for collisions evolving older planets. Second, and this is clearly the more interesting effect, older planets take much longer to recover from such impacts. For example, a $1 \mathrm{Gyr}$ old planet remains over-luminous for several $10^{9} \mathrm{yr}$, while a $10 \mathrm{Myr}$ planet becomes indistinguishable from the reference planet after a few $10^{7}$. While this long afterglow time potentially increases the detection probability of the planets considerably, the likelihood of such collisions occurring in a 1 Gyr old system is probably very low. Hence, we still believe that the best chances for detecting impacted planets are to search in young systems (age below $100 \mathrm{Myr}$ ).

\subsection{Detectability of post-impact EGPs}

To compute the detectability of this kind of impacted planet, we need to compute the flux received at Earth from similar sources. To do this, we follow Stern (1994) to assume that the planets radiate as black bodies and that they are typically located at a distance of $135 \mathrm{pc}$. The thermal flux received from the Earth is

$F_{\lambda}(T) \sim\left(\frac{R}{d}\right)^{2} B_{\lambda}(T) \lambda$

when $d$ is the distance, $B_{\lambda}(T)$ Planck's function, and $R$ the radius of the observed planet. We evaluate this flux for both the reference and the impacted planet (neglecting irradiation from the parent star) immediately after the short-term evolution phase and after 10 Myr. These fluxes are then compared with the one from a typical $\mathrm{G} 2 \mathrm{~V}$ star assumed to radiate as a black body with an effective temperature of $5780 \mathrm{~K}$. The corresponding results are shown in Fig. 21.

As expected, the impacted planets are indeed more luminous than their reference counterparts. However, when compared to the stellar luminosity, the increase remains quite small. The maximum of the emitted light is around $2.2 \mu \mathrm{m}$ for the post-impact planet (corresponding to the $K$ band), whereas this maximum moves to $\sim 4.8 \mu \mathrm{m}$ ( $M$ band) $10 \mathrm{Myr}$ after the impact.

Of course, it is well known that the presence of the host star renders the detection of the planet extremely difficult. This is best shown if we compute the flux contrast ratio between the planet and the star that we define by $f_{\lambda}=F(\lambda, p) / F(\lambda, *)$, where $F(\lambda, p)$ and $F(\lambda, *)$ are the fluxes at a wavelength $\lambda$ of the planet 


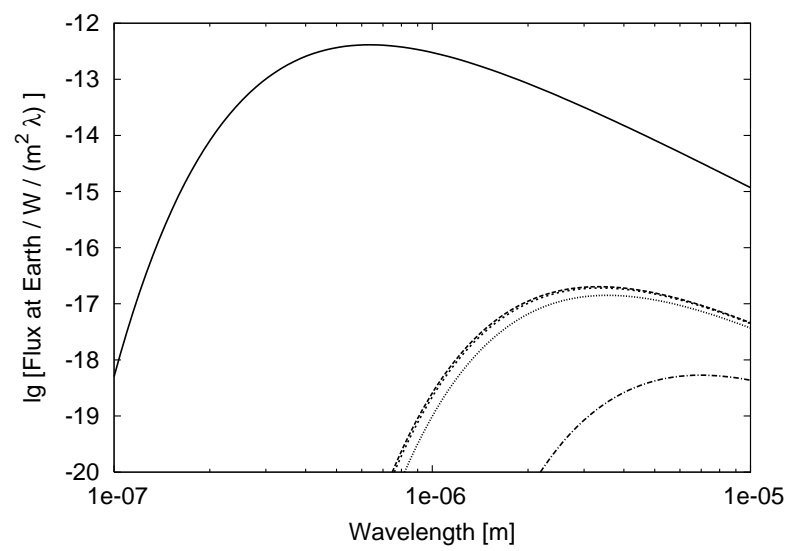

Fig. 21. Thermal fluxes from bodies located 135 pc from Earth. From top to bottom, first line: G2 V star; second line: post-impact planet (offaxis collision); third line: post-impact planet (head-on collision); fourthline: reference planet; and last line: planets $10 \mathrm{Myr}$ after a collision (all are indistinguishable).

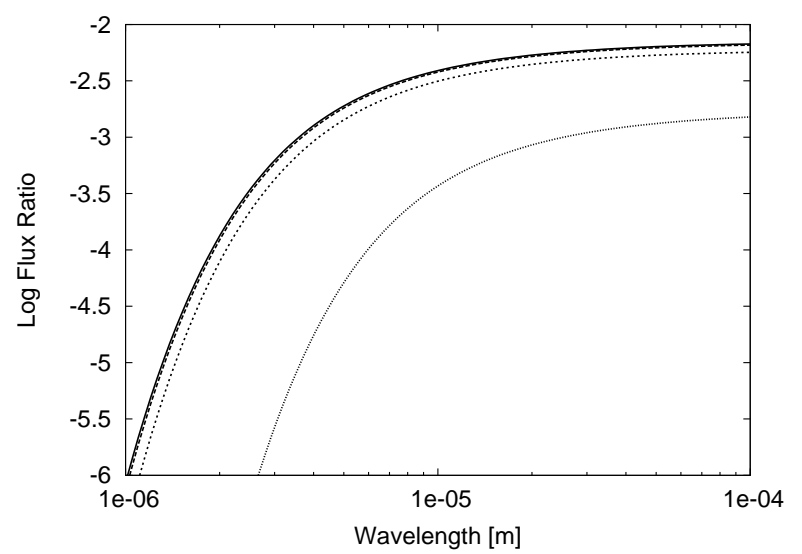

Fig. 22. Contrast ratios for the same planets as in Fig.21. The central star is again assumed to be a G2 V star, and the lines have the same meaning as in Fig. 21. From top to bottom, first lines corresponds to the post-impact planet from SIM4, whereas the last lines gives the ratio of the post-impact planet $10 \mathrm{Myr}$ after the collision to the G2 V star.

and the star, respectively. Figure 22 shows the flux contrast ratios of the same planets as in Fig. 21 - relative to a standard G2 V star. It is interesting to note that collisions increase the ratio marginally. On the other hand, the ratio of the young Jupiter is almost $f_{\lambda} \sim 0.01$ (in $I R$ band), which can be considered as the threshold for signal detection by photometric monitoring by ground based-telescopes (e.g. Borucki et al. 2001).

Finally, we can compute the number of stars, $N_{*}$, that we have to monitor in order to detect a luminous post-impact planet by using the expression

$N_{*}=\left(f_{\mathrm{p}} \tilde{N}_{\mathrm{p}}\right)^{-1} \frac{T_{\mathrm{acc}}}{\left\langle t_{\mathrm{hot}}(t) \times \exp \left(-t / T_{\mathrm{acc}}\right)\right\rangle_{T_{\text {system }}}}$

where we assume that the rate of giant collisions decays exponentially with a typical timescale $T_{\text {acc }}$, and the mean of $t_{\text {hot }}(t) \times \exp \left(-t / T_{\text {acc }}\right)$ is defined for the times below the mean

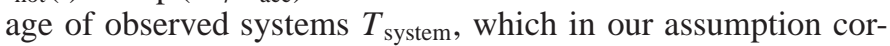
responds to the total lifetime during which collisions occur ${ }^{3}$. Here, $f_{\mathrm{p}}$ denotes the fraction of stars having planets $(\sim 0.01$ in

${ }^{3}$ Note that, due to the exponential decay in the denominator of Eq. (7), the precise value of $T_{\text {system }}$ is not important, being actually much higher than the typical accretion time $T_{\text {acc }}$. the pessimistic case and $\sim 0.3$ in the optimistic case), and $\tilde{N}_{\mathrm{p}}$ is the number of giant collisions involving giant planets per system. We adopt a typical Earth's accretion timescale of $\sim 40 \mathrm{Myr}$ for $T_{\text {acc }}$ (Wetherill 1990; Stern 1994). The mean age of the system, $T_{\text {system }}$, is assumed to amount to $\sim 100 \mathrm{Myr}$ (Stern 1994).

For the cooling timescale we use our estimate $t_{\text {hot }}=t_{\text {hot, } 2}$, and calculate $\tilde{t}_{\text {hot }, 2}=\left\langle t_{\text {hot }}(t) \times \exp \left(-t / T_{\text {acc }}\right)\right\rangle$ for the IR afterglows from the impacted $1 \mathrm{Myr}, 10 \mathrm{Myr}, 30 \mathrm{Myr}, 100 \mathrm{Myr}$, and 1 Gyr planets. We obtain $\tilde{t}_{\mathrm{hot}, 2}=35.7 \mathrm{Myr}^{4}$. Assuming typical values for $\tilde{N}_{\mathrm{p}}$ - the number of giant collisions involving giant planets in a given planetary system, of 1 to $10^{5}$, and assuming that $f_{\mathrm{p}}=0.01$, we find that roughly 1 in every $\sim 10-100$ systems with ages less than 100 Myr should harbor a planet in a post-impact stage for which the signature of the impact is still noticeable. We obtain the same order of magnitude by varying the accretion timescale $T_{\text {acc }}$ between 30 and $50 \mathrm{Myr}$.

For a given signal-to-noise ratio $(S / N)$, the integration time on the telescope for a background-dominated signal is given by (Bradt 2004)

$\langle t\rangle \sim \frac{1}{2}(S / N)^{2} \frac{\dot{S}_{\mathrm{s}}}{\left\langle\dot{S}_{\mathrm{p}}\right\rangle^{2}}$

where $\left\langle\dot{S}_{\mathrm{p}}\right\rangle=\pi / 4\left\langle j_{\mathrm{p}}\right\rangle D^{2}$ and $\dot{S}_{\mathrm{s}}=\pi / 4 j_{\mathrm{s}} D^{2}$ denote the number of photons stemming, respectively, from the planet and the parent star and falling on a detector of diameter $D$. Here, $\left\langle j_{\mathrm{p}}\right\rangle$ and $j_{\mathrm{s}}$ are the corresponding fluxes per square meter per second in a given spectral bandpass.

It can be easily calculated that the exposure time reduces roughly by factor 2.5 when comparing the signals from impacted and unimpacted planets. For example, observing an unimpacted 1 Myr old Jupiter located at a typical distance of $135 \mathrm{pc}$ with a Keck type telescope $(D=10 \mathrm{~m})$ and neglecting all other sources of noises associated with the telescope, would take about $100 \mathrm{~min}$ to reach $S / N=20$ compared to the 40 min needed to achieve a similar $S / N$ for an impacted planet under same condition. With the previous results for the number of scanned stars, we find out that it would take around $\sim 1-10$ nights $(8 \mathrm{~h}$ per night assumed) to detect at least one giant collision on young Jupiter. However, to confirm that we indeed have detected a post-impact planet, one should perform a second observation (Stern 1994), which requires additional efforts.

\section{Discussion and summary}

We have performed high-resolution SPH simulations, using realistic models of young Jupiter-like planets of different ages to calculate the energy-deposition profiles and the structure of postimpacted bodies. We have used these results to calculate the long term evolution of these planets. The effect of such giant collisions on the target planet in terms of flux increase and time scale to recover could also be derived, which allowed us to discuss the likelihood of detecting these objects.

Our calculations confirm some of the basic timescales derived by ZS03, in particular regarding the three first phases of the EM signal emitted during a giant impact. However, we found

${ }^{4}$ This latter value depends on the adopted value of the accretion timescale $T_{\text {acc }}$. Varying its value between 30 and $50 \mathrm{Myr}$, the corresponding $\tilde{t}_{\text {hot }, 2}$ varies between 25 and $45 \mathrm{Myr}$.

5 The higher value corresponds to the order of magnitude of such giant impacts necessary for accreting a mass similar to the one of Jupiter's core. 
major differences in the timescales of the IR afterglow: our simulations show that it has to be defined in another way than proposed by ZS03. Indeed, the impacted planet remains brighter than its unimpacted counterpart even after the whole energy deposited during the impact has been radiated away. Therefore, the timescale necessary for the impacted planet to reach a luminosity similar to its unimpacted counterpart is found to scale from $\sim 1 \mathrm{Myr}$ to $\sim 100 \mathrm{Myr}$, depending on the age of the impacted planet. This finally is reflected in an increase in the probability detection of post-impact planets.

Our calculations have shown that giant planets that have suffered from collisions are not orders of magnitude brighter than their unperturbed counterparts. Hence, even though the increase in luminosity reduces the required integration time by roughly a factor 2.5 , the fact that 1 in 10-100 planets is expected to be in a post-impact state keeps the search for these types of planets from being worthwhile for planet detection purposes unless the estimates of the collision frequency we have adopted here has been severely underestimated. The additional difficulty is that one has to provide powerful evidence that a post-impact planet has been detected, which requires the second observation. However, we note that detecting a planet in a post giant-impact phase would represent a major milestone in establishing the current ideas about planet formation. In this sense, it might still be worth looking for this type of object.

Finally, we note that the impact-induced changes in internal structure of giant planets result in larger planetary radii than otherwise predicted by theory. For some of the transiting exoplanets for which a radius determination is possible, there appears to be difference an unaccounted for between the observationally determined radius and the computed one. The case of HD 209458b (Charbonneau et al. 2000) with a mass $M=$ $0.69 \pm 0.02 M_{J}$ and radius $R=1.42_{-0.13}^{+0.10} R_{\mathrm{J}}$ is a well-known example of this kind of object. According to Baraffe et al. (2003), this radius is $\sim 20 \%$ larger than expected. We have shown that giant impacts involving an Earth-like planet can lead to a radius increase of roughly $7 \%$, which is not enough to account for the difference. In addition, given the age of HD 209458 of $5.5 \pm 1.5$ Gyr (Burrows et al. 2000) it is unlikely that the effect of a collision that occurred during the formation time of the system can still be seen today. Of course, we cannot exclude a giant impact involving a larger planet and occurring at later times, but we do not believe this to be the likely explanation.
Acknowledgements. The authors gratefully acknowledge partial support from the Swiss National Science Foundation.

\section{References}

Allard, F., Hauschildt, P. H., Alexander, D. R., Tamanai, A., \& Schweitzer, A. 2001, ApJ, 556, 357

Alexander, D. R., \& Ferguson, J. W. 1994, ApJ, 437, 879

Baraffe, I., Chabrier, G., Barman, T. S., et al. 2003, A\&A, 402, 701

Benz, W. 1990, SPH - A Review, in The Numerical Modelling of Nonlinear Stellar Pulsations, ed. B. J. Buchler, 269

Benz, W., Slattery, W. L., \& Cameron, A. G. W. 1986, Icarus, 66, 515

Benz, W., Slattery, W. L., \& Cameron, A. G. W. 1988, Icarus, 74, 516

Benz, W., Cameron, A. G. W., \& Melosh, H. J. 1989, Icarus, 81, 113

Borucki, W. J., Caldwell, D., Koch, D. G., et al. 2001, PASP, 113, 439

Bradt, H. 2004, Astronomy Methods (Cambridge University Press)

Burrows, A., Guillot, T., Hubbard, W. B., et al. 2000, ApJ, 534, 97

Canup, R. M., \& Aspahug, E. 2003, in 34th Annual Lunar and Planetary Science Conference, League City, Texas

Canup, R. M. 2004, Icarus, 168, 433

Canup, R. M. 2005, Science, 307, 5709, 546

Charbonneau, D., Brown, T. M., Latham, D. W. 2000, ApJ, 529, 45

Cody, A. M., \& Sasselov, D. D. 2002, ApJ, 569, 451

Dziewonski, A. M., \& Anderson, D. L. 1981, PEPI, 25, 297

Guillot, T., Chabrier, G. Morel, P., et al. 1994, Icarus, 112, 354

Korycansky, D. G., Zahnle, K. J., \& Mac Low, M.-M. 2000, Icarus, 146, 387

Lissauer, J. J., \& Safronov 1991, Icarus, 93, 288

Melosh, H. J. 1989, in Impact Cratering - a Geologic Process (New York: Oxford University Press)

Morris, J. P., \& Monaghan, J. J. 1997, J. Comput. Phys., 136, 41

Pritchard, M. E., \& Stevenson, D. J. 2000, in Origin of the earth and moon, ed. R. M. Canup, K. Righter and 69 collaborating authors (Tucson: University of Arizona Press), 179

Saumon, D., Chabrier, G., \& van Horn, H. M. 1995, ApJS, 99, 713

Saumon, D., \& Guillot, T. 2004, ApJ, 609, 1170

Slattery, W. L., Benz, W., \& Cameron, A. G. W. 1992, Icarus, 99, 167

Steinmetz, M., \& Müller, E. 1993, A\&A, 268, 391

Stern, A. S. 1992, ARA\&A, 30, 185

Stern, A. S. 1994, AJ, 108, 6

Stevenson, D. J. 1987, Ann. Rev. Earth Plan. Sci., 15, 271

Takata, T., O'Keefe, J. D., Ahrens, T. J., et al. 1994, Icarus, 109, 3

Thompson, S. L., \& Lauson, H. S. 1984, Improvement in the Chart D RadiationHydrodynamic Code. III. Revised Analytical Equations of State. Sandia Laboratories Report SC-RR-71 0714

Tonks, W. B., Melosh, H. J., \& McKinnon, W. B. 1990, LPI, 21, 1260

Wetherill, G. 1985, LPI, 16, 901

Wetherill, G. 1990, Ann. Rev. Earth Planet. Sci., 18, 205

Zhang, B., \& Sigurdsson, S. 2003, ApJ, 596, 95 